PROCEEDINGS OF THE

AMERICAN MATHEMATICAL SOCIETY

Volume 137, Number 7, July 2009, Pages 2335-2344

S 0002-9939(09)09839-6

Article electronically published on February 17, 2009

\title{
UNIQUENESS AND NON-UNIQUENESS IN INVERSE RADIATIVE TRANSFER
}

\author{
PLAMEN STEFANOV AND ALEXANDRU TAMASAN
}

(Communicated by Walter Craig)

\begin{abstract}
We characterize the non-uniqueness in the inverse problem for the stationary transport model, in which the absorption $a$ and the scattering coefficient $k$ of the media are to be recovered from the albedo operator. We show that "gauge equivalent" pairs $(a, k)$ yield the same albedo operator, and that we can recover uniquely the class of the gauge equivalent pairs. We apply this result to show unique determination of the media when the absorption $a$ depends on the line of travel through each point while the scattering $k$ obeys a symmetry property. Previously known results concerned the directional independent absorption $a$.
\end{abstract}

\section{INTRODUCTION}

This paper considers the problem of recovering the absorption and scattering properties of a bounded, convex medium $\Omega \subset \mathbb{R}^{n}, n \geq 3$ from the spatial-angular measurements of the density of particles at the boundary $\partial \Omega$. Provided that the particles interact with the medium but not with each other, the radiation transfer in the steady-state can be modeled by the transport equation

$$
-\theta \cdot \nabla u(x, \theta)-a(x, \theta) u(x, \theta)+\int_{S^{n-1}} k\left(x, \theta^{\prime}, \theta\right) u\left(x, \theta^{\prime}\right) d \theta^{\prime}=0,
$$

for $x \in \Omega$ and $\theta \in S^{n-1}$; see, e.g., [9, 22. The function $u(x, \theta)$ represents the density of particles at $x$ traveling in the direction $\theta, a(x, \theta)$ is the absorption coefficient at $x$ for particles traveling in the direction of $\theta$, and $k\left(x, \theta^{\prime}, \theta\right)$ is the scattering coefficient (or the collision kernel), which accounts for particles from an arbitrary direction $\theta^{\prime}$ that scatter in the direction of travel $\theta$.

The medium is probed with the given radiation

$$
\left.u\right|_{\Gamma_{-}}=f_{-},
$$

and the exiting radiation

$$
\left.u\right|_{\Gamma_{+}}=: \mathcal{A}\left[f_{-}\right]
$$

is detected, where

$$
\Gamma_{ \pm}=\left\{(x, \theta) \in \partial \Omega \times S^{n-1} \mid \quad \pm \theta \cdot n(x)>0\right\}
$$

Received by the editors September 15, 2008.

2000 Mathematics Subject Classification. Primary 35R30, 78A46.

The first author was partly supported by NSF FRG Grant No. 0554065.

(C)2009 American Mathematical Society Reverts to public domain 28 years from publication 
with $n(x)$ denoting the outer unit normal at a boundary point $x$. The equation (3) defines the albedo operator $\mathcal{A}$ which takes the incoming flux $f_{-}$to the outgoing flux $\left.u\right|_{\Gamma_{+}}$at the boundary.

In general, the boundary value problem (11) and (2) may not be uniquely solvable, but it has a unique solution for generic $(a, k)$; see [26] and Proposition 3.1 below. Unique solvability can also be obtained under some physically relevant subcritical conditions such as (6) or (7) below. We assume that $(a, k)$ is such that the direct problem (11) and (2) is well-posed.

The inverse boundary value problem of radiative transfer is to recover the absorption $a(x, \theta)$ and the scattering kernel $k\left(x, \theta^{\prime}, \theta\right)$ from knowledge of the albedo operator $\mathcal{A}$. One could also study the time-dependent version of (11), and then the kernel of $\mathcal{A}$ contains one more variable that gives us more information. This problem has been solved under some restrictive assumptions (e.g. $k$ of a special type or independent of a variable) in [1, 2, 3, 5, 16, 17, 18, 27, 28. In three or higher dimensions, uniqueness and reconstruction results for general $k$ and $a=a(x)$ were established in [11. The approach there is based on the study of the singularities of the fundamental solution of (11) (see also [8]), and the singularities of the Schwartz kernel of $\mathcal{A}$. Stability estimates for $k$ of special type were established in [24, 29, and recently, for general $k$, in [6]. Uniqueness and reconstruction results in a Riemannian geometry setting, including recovery of a simple metric, were established in 19. Similar results for the time-dependent model were established in [10, and in 13 for the Riemannian case. In planar domains the work in 25 shows stable determination of the isotropic absorption and small scattering, and extensions to simple Riemannian geometry are given in [20. Also in two-dimensional domains we point out that the recovery of $k$ is only known under smallness conditions which are more restrictive than what is needed to solve the direct problem, e.g., more restrictive than (6) or (7) below. On the other hand, in the time-dependent case, the extra variable allows us to treat the planar case without such restrictions; see [10. We also mention here the recent works [7, 14, 15, in which the coefficients are recovered from angularly averaged measurements rather than from knowledge of the whole albedo operator $\mathcal{A}$.

The above-mentioned results concern media with directionally independent absorption properties; for transport with variable velocity $v$, which now belongs to an open subset of $\mathbb{R}^{n}$, the absorption may depend on the speed $a=a(x,|v|)$.

In general, in media with an anisotropic absorption, the albedo operator does not determine the coefficients uniquely. For example, if $k \equiv 0$ the obstruction to unique determination can be readily seen. The most one can recover from the albedo operator $\mathcal{A}$ are the integrals $\int_{\mathbb{R}} a(x+t \theta, \theta) d t$; see [11. In [6], they are shown to be stably recovered independently of $k$. In other words, for each fixed direction $\theta \in S^{n-1}$, we know the integral of the map $a(\cdot, \theta)$ over the parallel lines in the direction of $\theta$. This is insufficient data, since one can smoothly change the $x$-variable in the direction of $\theta$ while preserving the integral; see also the theorem below.

The non-uniqueness described above and the need to assume that $a$ is isotropic left the theory of the inverse radiative problem in a somewhat unsatisfactory state. It was not clear whether the uniqueness failed if $k \not \equiv 0$; neither was it known what information about $(a, k)$ can still be recovered. The purpose of this work is to fill this gap. We show that a certain family of "gauge transformations" of $(a, k)$ does 
not change the albedo operator $\mathcal{A}$ and that, given $\mathcal{A}$, one can recover uniquely the class of gauge equivalent pairs $(a, k)$. The recovery of the gauge equivalent class is explicit, as the recovery of $a=a(x)$ and $k$ in [1] is explicit.

\section{MAIN RESULTS}

The pair $(a, k)$ is assumed to satisfy the admissibility condition

$$
\sup _{(x, \theta) \in \Omega \times S^{n-1}}\left(|a(x, \theta)|+\int_{S^{n-1}}\left|k\left(x, \theta, \theta^{\prime}\right)\right| d \theta^{\prime}\right)<\infty .
$$

Let $T$ be the operator defined by the l.h.s. of (11). For $(a, k)$ admissible, the second and the third terms of $T$ are bounded operators on $L^{1}\left(\Omega \times S^{n-1}\right)$, while the first term is unbounded. We view $T$ as an unbounded operator on $L^{1}\left(\Omega \times S^{n-1}\right)$ with the domain

$$
D(T)=\left\{f \in L^{1}\left(\Omega \times S^{n-1}\right)\left|\theta \cdot \nabla f \in L^{1}\left(\Omega \times S^{n-1}\right), f\right|_{\Gamma_{-}}=0\right\} ;
$$

see also [11. Since the direct problem (11) and (2) can always be reduced to a non-homogeneous problem with a homogeneous boundary condition, invertibility of $T$ implies well-posedness. We say that the direct problem is well-posed if $T^{-1}$ exists as a bounded operator.

As an example, we have the following two subcritical conditions that yield wellposedness; see, e.g., 6, 11, 12, 21, 22 and Proposition 3.1 below. Either

$$
\sup _{(x, \theta) \in \Omega \times S^{n-1}}\left|\tau(x, \theta) \int_{S^{n-1}} k\left(x, \theta, \theta^{\prime}\right) d \theta^{\prime}\right|<1,
$$

where $\tau(x, \theta)$ is the total free path of a particle at $(x, \theta)$ (see the beginning of the next section), or

$$
a(x, \theta)-\int_{S^{n-1}} k\left(x, \theta, \theta^{\prime}\right) d \theta^{\prime} \geq 0, \text { a.e. } \Omega \times S^{n-1} .
$$

We start with a simple observation that seems to be new. We will show that there is non-uniqueness even if $k \not \equiv 0$, too. Let $\phi(x, \theta)>0$ be such that $\phi=1$ on $\Gamma:=\partial \Omega \times S^{n-1}$. Set $\widetilde{a}=a-\theta \cdot \nabla_{x} \log \phi$. Then we can rewrite (10) as

$$
-\theta \cdot \nabla_{x} u-\phi^{-1}\left(\theta \cdot \nabla_{x} \phi\right) u-\widetilde{a} u+\int_{S^{n-1}} k\left(\cdot, \theta^{\prime}, \theta\right) u\left(\cdot, \theta^{\prime}\right) d \theta^{\prime}=0 .
$$

Multiply by $\phi$ to get

$$
-\theta \cdot \nabla_{x}(\phi u)-\widetilde{a} \phi u+\phi \int_{S^{n-1}} k\left(\cdot, \theta^{\prime}, \cdot\right) u\left(\cdot, \theta^{\prime}\right) d \theta^{\prime}=0 .
$$

The function $\widetilde{u}=\phi u$ thus solves (10) with $(a, k)$ replaced by $(\widetilde{a}, \widetilde{k})$, where those two pairs are related by the "gauge transformation"

$$
\widetilde{a}=a-\theta \cdot \nabla_{x} \log \phi, \quad \widetilde{k}\left(x, \theta^{\prime}, \theta\right)=\frac{\phi(x, \theta)}{\phi\left(x, \theta^{\prime}\right)} k\left(x, \theta^{\prime}, \theta\right) .
$$

Since $\phi=1$ on $\Gamma$, the boundary data do not change. Therefore, if $\widetilde{\mathcal{A}}$ is the albedo operator corresponding to the pair $(\widetilde{a}, \widetilde{k})$, then $\mathcal{A}=\widetilde{\mathcal{A}}$.

Our main result is that this is the only obstruction to non-uniqueness. 
Theorem 2.1. Let $(a, k)$ and $(\widetilde{a}, \widetilde{k})$ be two admissible pairs for which the direct problem is well-posed, and let $\mathcal{A}$ and $\widetilde{\mathcal{A}}$ be the corresponding albedo operators. Then $\mathcal{A}=\widetilde{A}$ if and only if there exists a positive $\phi \in L^{\infty}\left(\Omega \times S^{n-1}\right)$ with $\theta \cdot \nabla_{x} \phi(x, \theta) \in$ $L^{\infty}\left(\Omega \times S^{n-1}\right)$ and $\phi=1$ for $x \in \partial \Omega$ so that (8) holds.

The proof of the theorem is based on the analysis of the singularities of the Schwartz kernel of $\mathcal{A}$, as in [11. Recall that $n \geq 3$ here. On the other hand, if $n=2$, we can still recover $a$ up to a gauge transformation; see also Section 6 ,

Theorem 2.1 allows us to obtain a few new uniqueness results under additional conditions. One of them concerns the case where we have anisotropic media with absorption $a(x, \theta)$ that depends on the line of travel through each point (but not on the direction):

$$
a(x, \theta)=a(x,-\theta), x \in \Omega, \theta \in S^{n-1},
$$

and a scattering coefficient $k>0$ satisfying the following symmetry condition:

$$
k\left(x, \theta, \theta^{\prime}\right)=k\left(x, \theta^{\prime}, \theta\right), x \in \Omega, \theta, \theta^{\prime} \in S^{n-1} .
$$

Corollary 2.2. Let $(a, k),(\widetilde{a}, \widetilde{k})$ be two admissible and subcritical pairs which yield the same albedo operator. Assume that $k$ and $\widetilde{k}>0$ satisfy (10).

(i) Then $k=\widetilde{k}$ and $a=\tilde{a}+\theta \cdot \nabla v(x)$ for some function $v(x)$ vanishing on $\partial \Omega$. In particular, one can recover the total absorption at a.e. $x$, i.e., $\int a(x, \theta) d \theta=$ $\int \widetilde{a}(x, \theta) d \theta$.

(ii) If, in addition, $a$ and $\widetilde{a}$ satisfy (9), then $a=\widetilde{a}$.

Note that any two pairs as in (i) yield the same albedo operator, so this is the most we can say in this case. The symmetry assumption (10) occurs naturally in some models of optical tomography, where the scattering of light in a tissue depends on the angle between the two directions: $k\left(x, \theta, \theta^{\prime}\right)=k\left(x, \theta \cdot \theta^{\prime}\right)$.

One can formulate and prove similar results in the case where the velocity belongs to an open subspace of $\mathbb{R}^{n}$; i.e., the speed can change, as in 11. We restrict ourselves to the fixed speed case $(|\theta|=1)$ for the sake of simplicity of the exposition. Also, the fixed speed model is the one that is most often discussed in the literature.

\section{Preliminaries}

In this section we recall some results from [11] recast to the one-speed velocities and introduce notation.

Let $\tau_{ \pm}(x, \theta)$ be the travel time it takes a particle at $x$ to reach the boundary while moving in the direction of $\pm \theta$ and define $\tau(x, \theta)=\tau_{-}(x, \theta)+\tau_{+}(x, \theta)$. Since we work with unit-speed velocities, note that $\tau(x, \theta) \leq \operatorname{diam}(\Omega)$. Let $d \xi(x, \theta)=$ $|n(x) \cdot \theta| d \mu(x) d \theta$, where $d \mu(x)$ is the induced Lebesgue measure on the boundary and $d \theta$ is the normalized measure on the sphere. Also let $\delta_{\{x\}}\left(x^{\prime}\right)$ represent the delta distribution with respect to the boundary measure $d \mu\left(x^{\prime}\right)$ supported at $x \in \partial \Omega$, and let $\delta_{\{\theta\}}\left(\theta^{\prime}\right)$ represent the delta distribution with respect to $d \theta$ centered at $\theta \in S^{n-1}$.

Also, for $(x, \theta) \in \Omega \times S^{n-1}$, let $x_{\theta}^{+}$denote the exiting point if traveling from $x$ in the $\theta$-direction, and let $x_{\theta^{\prime}}^{-}$be the entrance point at the boundary to reach the inside point $x$ by traveling in the $\theta^{\prime}$-direction, i.e.

$$
x_{\theta}^{+}:=x+\tau_{+}(x, \theta) \text { and } x_{\theta^{\prime}}^{-}:=x-\tau_{-}\left(x, \theta^{\prime}\right) \theta^{\prime} .
$$

For the proposition below, we introduce the class of regular scattering kernels $k \in C\left(\bar{\Omega}, L^{\infty}\left(S^{n-1}, L^{1}\left(S^{n-1}\right)\right)\right)$. Then the map $\int k\left(x, \theta^{\prime}, \theta\right) \phi\left(\theta^{\prime}\right) d \theta^{\prime}$ is bounded on 
$L^{1}\left(S^{n-1}\right)$ continuously depending on $x$. Our notion of regular $k$ is stronger than that in [21], and, in particular, it allows us to use the results there.

Proposition 3.1. The direct problem is well-posed; i.e., T has a bounded inverse on $L^{1}\left(\Omega \times S^{n-1}\right)$ if either (6) or (77) holds. Moreover, the direct problem is well-posed for an open dense set of $(a, k) \in L^{\infty}\left(\Omega \times S^{n-1}\right) \times C\left(\bar{\Omega}, L^{\infty}\left(S^{n-1}, L^{1}\left(S^{n-1}\right)\right)\right)$.

Proof. We will first discuss the well-posedness under the conditions (6) or (7). Assume (6) first. By [11, Proposition 2.3], and since $\tau$ is bounded, we get that $T^{-1}$ is bounded. The subcritical case (7) is covered in [6, Section 2].

The generic statement is proven in $L^{2}\left(\Omega \times S^{n-1}\right)$ for $C^{2}$ coefficients in 26]. In the $L^{1}$ spaces under consideration, we proceed in a similar way. Let $K$ be the integral operator in (11) and let $T_{1}=T-K$. Then $K T_{1}^{-1} K$ is weakly compact in $L^{1}\left(\Omega \times S^{n-1}\right)$; see [21]. Therefore, $\left(K T_{1}^{-1}\right)^{2}$ is weakly compact, and its square is compact. For a fixed $a$, consider the family $\lambda k$, where $\lambda$ is a real parameter. By the analytic Fredholm alternative in Banach spaces [23], $\lambda \mapsto\left(I-\left(\lambda K T_{1}^{-1}\right)^{4}\right)^{-1}$ is a meromorphic family. This implies that $\lambda \mapsto\left(I-\lambda K T_{1}^{-1}\right)^{-1}$ is also meromorphic, and thus $T^{-1}=\left(I-\lambda K T_{1}^{-1}\right)^{-1} T_{1}^{-1}$ exists for all but a discrete set of $\lambda$ 's. This shows that there is a dense set of pairs yielding a well-posed problem. The fact that this set is also open follows from a perturbation argument around each $(a, k)$ for which $T^{-1}$ is bounded; thus $\left(I-\lambda K T_{1}^{-1}\right)^{-1}$ corresponding to nearby pairs exists.

Note that one can set $a(x, \theta)=a_{0}(x, \theta)+\int k\left(x, \theta, \theta^{\prime}\right) d \theta^{\prime}$, where the integral represents the attenuation due to the change of direction, while $a_{0}$ is the absorption. Then one can prove in the same way that the direct problem is well-posed for generic $\left(a_{0}, k\right)$, and moreover, for any $a_{0}$, this is true for generic $k$ 's.

Proposition 3.2. Assume that the direct problem is well-posed. Then the albedo operator $\mathcal{A}: L^{1}\left(\Gamma_{-}, d \xi\right) \rightarrow L^{1}\left(\Gamma_{+}, d \xi\right)$ is bounded and its Schwartz kernel $\alpha$ is given by $\alpha=\alpha_{1}+\alpha_{2}+\alpha_{3}$, where

$$
\begin{aligned}
\alpha_{1}\left(x, \theta, x^{\prime}, \theta^{\prime}\right)= & e^{\int_{0}^{\tau_{-}(x, \theta)} a(x-t \theta, \theta) d t} \delta_{\left\{x_{\theta}^{-}\right\}}\left(x^{\prime}\right) \delta_{\{\theta\}}\left(\theta^{\prime}\right), \\
\alpha_{2}\left(x, \theta, x^{\prime}, \theta^{\prime}\right)= & \int_{0}^{\tau_{-}(x, \theta)} e^{-\int_{0}^{s} a(x-t \theta, \theta) d t} e^{-\int_{0}^{\tau_{-}\left(x-s \theta, \theta^{\prime}\right)} a\left(x-s \theta-t \theta^{\prime}, \theta^{\prime}\right) d t} \\
& \times k\left(x-s \theta, \theta^{\prime}, \theta\right) \delta_{\left\{x-s \theta-\tau_{-}\left(x-s \theta, \theta^{\prime}\right) \theta^{\prime}\right\}}\left(x^{\prime}\right) d s, \\
\left|n\left(x^{\prime}\right) \cdot \theta^{\prime}\right|^{-1} \alpha_{3} \in & L^{\infty}\left(\Gamma_{-} ; L^{1}\left(\Gamma_{+}, d \xi\right)\right) .
\end{aligned}
$$

This proposition is formulated in 11 under the assumption that the system is subcritical; i.e., either (6) or (77) holds. We remark that those conditions are only used in the proof in the analysis of $\alpha_{3}$ to guarantee that $T^{-1}$ exists in $L^{1}$, something that we assume here.

Let $\phi \in C_{0}^{\infty}(B(0 ; 1))$ with $0 \leq \phi \leq 1$ and $\phi \equiv 1$ near the origin be a cut-off function. Given $\epsilon>0$ we define for $x, x^{\prime} \in \mathbb{R}^{n}$ and $\theta, \theta^{\prime} \in S^{n-1}$ :

$$
\phi_{\epsilon}\left(x, \theta, x^{\prime}, \theta^{\prime}\right)=\phi\left(\frac{x_{\theta}^{-}-x^{\prime}}{\epsilon}\right) \phi\left(\frac{\theta-\theta^{\prime}}{\epsilon}\right) .
$$


Proposition 3.3. Assume that the direct problem is well-posed. Then the limit below holds in $L^{1}\left(\Gamma_{+}, d \xi\right)$ :

$$
\lim _{\epsilon \rightarrow 0} \int_{\Gamma_{-}} \alpha\left(x, \theta, x^{\prime}, \theta^{\prime}\right) \phi_{\epsilon}\left(x, \theta, x^{\prime}, \theta^{\prime}\right) d \mu\left(x^{\prime}\right) d \theta=e^{-\int_{-\infty}^{\infty} a(x+t \theta, \theta) d t}>0 \text { a.e. } \Gamma_{+} \cdot
$$

For linearly independent $\theta, \theta^{\prime} \in S^{n-1}$, let $\pi_{\theta, \theta^{\prime}}(x)$ denote the projection of $x$ onto the plane through the origin spanned by $\theta$ and $\theta^{\prime}$. Let $\theta_{\perp}^{\prime}$ be a unit vector in $\operatorname{span}\left\{\theta, \theta^{\prime}\right\}$ orthogonal to $\theta^{\prime}: \theta_{\perp}^{\prime} \cdot \theta^{\prime}=0$. Let $\varphi \in C_{0}^{\infty}(-1,1)$ with $0 \leq \varphi \leq 1$ and $\int_{\mathbb{R}} \varphi(t) d t=1$ be a cut-off function in $\mathbb{R}$ and $\phi$ be the cut-off function in $\mathbb{R}^{n}$ introduced earlier. Define the test function

$$
\phi_{\epsilon, \delta}\left(x ; \theta, \theta^{\prime}\right)=\frac{1}{\epsilon} \varphi\left(\frac{x \cdot \theta_{\perp}^{\prime}}{\epsilon \theta \cdot \theta_{\perp}^{\prime}}\right) \phi\left(\frac{x-\pi_{\theta, \theta^{\prime}}(x)}{\delta}\right) .
$$

With $x_{\theta}^{+}$and $x_{\theta^{\prime}}^{-}$given by (11) and $\phi_{\epsilon, \delta}$ given by (17), we define in $\Omega \times S^{n-1} \times S^{n-1}$ :

$$
I_{\epsilon, \delta}\left(x, \theta^{\prime}, \theta\right):=\int_{\partial \Omega} \alpha\left(x_{\theta}^{+}, \theta, x^{\prime}, \theta^{\prime}\right) \phi_{\epsilon, \delta}\left(x^{\prime}-x_{\theta^{\prime}}^{-}, \theta, \theta^{\prime}\right) d \mu\left(x^{\prime}\right) .
$$

Proposition 3.4. Assume that the direct problem is well-posed. Then the following limit holds in $L^{1}\left(\Omega ; L_{l o c}^{1}\left(I^{\sharp}\right)\right)$ :

$$
I_{0,0}\left(x, \theta^{\prime}, \theta\right):=\lim _{\epsilon \rightarrow 0} \lim _{\delta \rightarrow 0} I_{\epsilon, \delta}\left(x, \theta^{\prime}, \theta\right)=e^{-\int_{-\infty}^{0} a\left(x+t \theta^{\prime}, \theta^{\prime}\right) d t-\int_{0}^{\infty} a(x+t \theta, \theta) d t} k\left(x, \theta^{\prime}, \theta\right),
$$

where $I^{+}:=\left\{\left(\theta, \theta^{\prime}\right) \in S^{n-1} \times S^{n-1}: \theta^{\prime} \neq \pm \theta\right\}$.

\section{Proof of Theorem 2.1 and Corollary 2.2}

Proof of Theorem 2.1. From Proposition 3.3, we can recover

$$
\int_{-\infty}^{\infty} a(x+s \theta, \theta) d s, \quad(x, \theta) \in \Omega \times S^{n-1} .
$$

In particular, the integrals above for $f=a-\widetilde{a}$ vanish:

$$
\int_{-\infty}^{\infty} f(x+s \theta, \theta) d s=0, \quad \forall(x, \theta) \in \Omega \times S^{n-1} .
$$

The kernel of the linear transform (20) is easy to describe. Set

$$
v(x, \theta)=\int_{-\infty}^{0} f(x+s \theta, \theta) d s, \quad(x, \theta) \in \Omega \times S^{n-1} .
$$

Then $v \in L^{\infty}\left(\Omega \times S^{n-1}\right)$ with $\theta \cdot \nabla_{x} v=f \in L^{\infty}\left(\Omega \times S^{n-1}\right)$ and $v=0$ on $\Gamma_{-}$. From (21) we also get that $v=0$ on $\Gamma_{+}$; therefore $v(x, \theta)=0$ for $x \in \partial \Omega$ and a.e. $\theta \in S^{n-1}$.

Set

$$
\phi(x, \theta)=e^{v(x, \theta)}
$$

to get $a-\widetilde{a}=\theta \cdot \nabla_{x} \log \phi$ as claimed. This shows the first part of (8).

Now, from Proposition 3.4 we get

$$
e^{-\int_{-\infty}^{0} a\left(x+t \theta^{\prime}, \theta^{\prime}\right)-\int_{0}^{\infty} a(x+t \theta, \theta)} k\left(x, \theta^{\prime}, \theta\right)=e^{-\int_{-\infty}^{0} \widetilde{a}\left(x+t \theta^{\prime}, \theta^{\prime}\right)-\int_{0}^{\infty} \widetilde{a}(x+t \theta, \theta)} \widetilde{k}\left(x, \theta^{\prime}, \theta\right) .
$$


Using the first equality in (8), which we have already proved, after a simple calculation, we derive

$$
\widetilde{k}\left(x, \theta^{\prime}, \theta\right)=\frac{\phi(x, \theta)}{\phi\left(x, \theta^{\prime}\right)} k\left(x, \theta^{\prime}, \theta\right)
$$

Proof of Corollary 2.2. Next we use the characterization above to prove uniqueness in the symmetric case. By swapping $\theta$ and $\theta^{\prime}$ in (24) we get

$$
\begin{aligned}
\widetilde{k}\left(x, \theta, \theta^{\prime}\right) & =\frac{\phi\left(x, \theta^{\prime}\right)}{\phi(x, \theta)} k\left(x, \theta, \theta^{\prime}\right)=\frac{\phi\left(x, \theta^{\prime}\right)}{\phi(x, \theta)} k\left(x, \theta^{\prime}, \theta\right) \\
& =\frac{\phi\left(x, \theta^{\prime}\right)^{2}}{\phi(x, \theta)^{2}} \widetilde{k}\left(x, \theta^{\prime}, \theta\right)=\frac{\phi\left(x, \theta^{\prime}\right)^{2}}{\phi(x, \theta)^{2}} \widetilde{k}\left(x, \theta, \theta^{\prime}\right) .
\end{aligned}
$$

For the second and fourth equalities we used (10), while for the third one we used (24). Since $\widetilde{k}$ does not vanish, we conclude that $\phi\left(x, \theta^{\prime}\right)=\phi(x, \theta)$ for all $\theta, \theta^{\prime} \in S^{n-1}$. Therefore $\phi=\phi(x)$ is independent of $\theta$ and applying (24) again we conclude that $k=\widetilde{k}$.

So far we have shown that $a(x, \theta)-\widetilde{a}(x, \theta)=\theta \cdot \nabla_{x} \phi(x)$. If the symmetry relation (9) holds, then $a(x, \theta)-\widetilde{a}(x, \theta)=-\theta \cdot \nabla_{x} \phi(x)$. Therefore $a-\widetilde{a}=0$.

\section{Reconstruction formulas in the Symmetric CASE}

We showed in the previous section that under the symmetry hypotheses (10) and (9) there is uniqueness for $a, k$. The proof is constructive, but it still leads to the problem of recovering $a(x, \theta)$ first, up to $\theta \cdot \nabla \log \phi$, from its underdetermined $\mathrm{X}$-ray transform; see the l.h.s. of (21). In this section, we show that under the same assumptions, there is an explicit reconstruction of a different, simpler type. Namely, first we recover in (21) the integrals that measure the total absorption along straight lines, but we are not trying to determine $a$ from them. Then by (13) we recover

$$
e^{-\int_{-\infty}^{0} a\left(x+t \theta^{\prime}, \theta^{\prime}\right) d t-\int_{0}^{\infty} a(x+t \theta, \theta) d t} k\left(x, \theta^{\prime}, \theta\right) ;
$$

see (28) below. We do not know the attenuation term above because we know the attenuation along straight but not broken lines. We can however swap $\theta$ and $\theta^{\prime}$ and multiply the results. Then we get $k^{2}$ multiplied by the attenuation along the two lines through $x$ parallel to $\theta$, and $\theta^{\prime}$, respectively, and we know that attenuation. This recovers $k$, and then we recover $a$.

We need to strengthen the regularity assumptions on the coefficients to

$$
a \in C\left(\Omega \times S^{n-1}\right), k \in C\left(\Omega \times S^{n-1} \times S^{n-1}\right) .
$$

Firstly, we extend the limit in Proposition 3.3 valid for maps in $\Gamma_{+}$to maps in $\Omega \times S^{n-1}$. For $(x, \theta) \in \Omega \times S^{n-1}$ and $\epsilon>0$, let us denote

$$
J_{\epsilon}(x, \theta):=\int_{\Gamma_{-}} \alpha\left(x_{\theta}^{+}, \theta, x^{\prime}, \theta^{\prime}\right) \phi_{\epsilon}\left(x, \theta, x^{\prime}, \theta^{\prime}\right) d \mu\left(x^{\prime}\right) d \theta^{\prime},
$$

where $x_{\theta}^{+}$is given in (11). We remark here that $J_{\epsilon}$ is constant with $x$ varying in the direction of $\theta$; i.e. $J_{\epsilon}(x+t \theta, \theta)=J_{\epsilon}(x, \theta)$ for all $t$. 
Lemma 5.1. The limit below holds in $L^{1}\left(\Omega \times S^{n-1} ; \tau(x, \theta)^{-1} d x d \theta\right)$ :

$$
J_{0}(x, \theta):=\lim _{\epsilon \rightarrow 0} J_{\epsilon}(x, \theta)=\exp \left(-\int_{-\infty}^{\infty} a(x+t \theta, \theta) d t\right)>0 \text { a.e. } \Omega \times S^{n-1} .
$$

Proof. We use the change of variable formula

$$
\int_{\Omega \times S^{n-1}} f(x, \theta) d x d \theta=\int_{\Gamma_{+}} \int_{0}^{\tau_{-}\left(x^{+}, \theta\right)} f\left(x^{+}-s \theta\right) d s d \xi\left(x^{+}, \theta\right)
$$

for $f(x, \theta)=\left|J_{\epsilon}(x, \theta)-\exp \left(\int_{-\infty}^{\infty} a(x+t \theta, \theta) d t\right)\right| \tau(x, \theta)^{-1}$ to estimate

$$
\begin{aligned}
& \int_{\Omega \times S^{n-1}}\left|J_{\epsilon}(x, \theta)-\exp \left(\int_{-\infty}^{\infty} a(x+t \theta, \theta) d t\right)\right| \tau(x, \theta)^{-1} d x d \theta \\
& =\int_{\Gamma_{+}} \int_{0}^{\tau_{-}\left(x^{+}, \theta\right)} \frac{\left|J_{\epsilon}\left(x^{+}-s \theta, \theta\right)-e^{\left(\int_{-\infty}^{\infty} a\left(x^{+}-s \theta+t \theta, \theta\right) d t\right)}\right|}{\tau\left(x^{+}-s \theta, \theta\right)} d s d \xi\left(x^{+}, \theta\right) \\
& =\int_{\Gamma_{+}}\left|J_{\epsilon}\left(x^{+}, \theta\right)-\exp \left(\int_{-\infty}^{\infty} a\left(x^{+}+t \theta, \theta\right) d t\right)\right| d \xi\left(x^{+}, \theta\right) .
\end{aligned}
$$

The last identity holds due to the fact that $\tau\left(x^{+}-s \theta, \theta\right)=\tau\left(x^{+}, \theta\right)=\tau_{-}\left(x^{+}, \theta\right)$ and that the integrand is constant in $s$. An application of Proposition 3.3 finishes the proof.

Following from Proposition 3.4 for a.e. $\left(x, \theta, \theta^{\prime}\right) \in \Omega \times I^{\text {H }}$ we get that

$$
\begin{aligned}
& e^{-\int_{-\infty}^{0} a\left(x+t \theta^{\prime}, \theta^{\prime}\right) d t-\int_{0}^{\infty} a(x+t \theta, \theta) d t} k\left(x, \theta^{\prime}, \theta\right)=I_{0,0}\left(x, \theta^{\prime}, \theta\right), \\
& e^{-\int_{-\infty}^{0} a(x+t \theta, \theta) d t-\int_{0}^{\infty} a\left(x+t \theta^{\prime}, \theta^{\prime}\right) d t} k\left(x, \theta, \theta^{\prime}\right)=I_{0,0}\left(x, \theta, \theta^{\prime}\right) .
\end{aligned}
$$

Using the symmetry assumption (10) on $k$, by multiplication of (28) and (29) we obtain

$$
e^{-\int_{-\infty}^{\infty} a\left(x+t \theta^{\prime}, \theta^{\prime}\right) d t-\int_{-\infty}^{\infty} a(x+t \theta, \theta) d t} k^{2}\left(x, \theta^{\prime}, \theta\right)=I_{0,0}\left(x, \theta^{\prime}, \theta\right) I_{0,0}\left(x, \theta, \theta^{\prime}\right) .
$$

Now using Lemma 5.1 we recover

$$
k\left(x, \theta^{\prime}, \theta\right)=\left(\frac{I_{0,0}\left(x, \theta^{\prime}, \theta\right) I_{0,0}\left(x, \theta, \theta^{\prime}\right)}{J_{0}(x, \theta) J_{0}\left(x, \theta^{\prime}\right)}\right)^{\frac{1}{2}} .
$$

Using the formula (31) in (29) we get for a.e. $\left(x, \theta, \theta^{\prime}\right) \in \Omega \times I^{*}$ that

$$
\int_{-\infty}^{0} a(x+t \theta, \theta) d t+\int_{0}^{\infty} a\left(x+t \theta^{\prime}, \theta^{\prime}\right) d t=\frac{1}{2} \log \left(\frac{I_{0,0}\left(x, \theta^{\prime}, \theta\right)}{I_{0,0}\left(x, \theta, \theta^{\prime}\right) J_{0}(x, \theta) J_{0}\left(x, \theta^{\prime}\right)}\right) .
$$

The continuity assumption (25) implies that the identity above extends pointwise in $\Omega \times S^{n-1} \times S^{n-1}$. By looking at backscattering $\theta^{\prime}=-\theta$ and making one change of variables $t \leftrightarrow-t$, we obtain

$$
\int_{-\infty}^{0} a(x+t \theta, \theta) d t+\int_{-\infty}^{0} a(x+t \theta,-\theta) d t=\frac{1}{2} \log \left(\frac{I_{0,0}(x,-\theta, \theta)}{I_{0,0}(x, \theta,-\theta) J_{0}(x, \theta) J_{0}(x,-\theta)}\right) .
$$


The left-hand side is differentiable in $x$ in the direction of $\theta$; hence so is the righthand side. By differentiating (33) in the direction of $\theta$ we obtain

$$
a(x, \theta)+a(x,-\theta)=\frac{1}{2} \theta \cdot \nabla_{x} \log \left(\frac{I_{0,0}(x,-\theta, \theta)}{I_{0,0}(x, \theta,-\theta) J_{0}(x, \theta) J_{0}(x,-\theta)}\right) .
$$

Since the absorption depends on the line through $x$, we have $a(x, \theta)=a(x,-\theta)$ and thus it can be recovered from the formula:

$$
a(x, \theta)=\frac{1}{4} \theta \cdot \nabla_{x} \log \left(\frac{I_{0,0}(x,-\theta, \theta)}{I_{0,0}(x, \theta,-\theta) J_{0}(x, \theta) J_{0}(x,-\theta)}\right) .
$$

\section{REMARKS}

6.1. The isotropic absorption case. The previously known uniqueness result 11 for isotropic absorption follows from Theorem 2.1 combined with the injectivity of the X-ray transform. If $f=a-\widetilde{a}$ depends on the position only, then (21) implies $f=0$. From the definition (23) we get $\phi \equiv 1$, which by (8) yields $k=\widetilde{k}$.

6.2. Other conditions that imply uniqueness. Assuming (10), we can only recover $a$ up to $\theta \cdot \nabla v(x)$ as above. For a unique recovery, the condition (9) suffices, but it can be replaced by something weaker. For example, we may require that $a$ is orthogonal to all such functions w.r.t. some measure $d \mu(\theta)$. This is equivalent to

$$
\int_{S^{n-1}} \theta \cdot \nabla_{x} a(x, \theta) d \mu(\theta)=0 .
$$

Note that the symmetry assumption (9) implies such a condition if $d \mu(\theta)$ is even.

6.3. The two-dimensional case. Since Proposition 3.3 is also valid in two dimensions, the proof of Theorem 2.1] shows that planar attenuations can still be recovered up to the gauge transformation in (8).

\section{ACKNOWLEDGEMENT}

This work started during the Summer School on Inverse Problems in Radiative Transfer at the University of California at Merced. The authors thank Arnold Kim for his kind hospitality.

\section{REFERENCES}

[1] D. S. Anikonov, The unique determination of the coefficients and the right side of the transport equation, Differential Equations 11(1975), pp. 6-12. MR0371321 (51:7540)

[2] _ Multidimensional inverse problems for the transport equation, Differential Equations 20(1984), pp. 608-614. MR747361 (86f:35171)

[3] Yu. Anikonov And B. A. Bubnov, Inverse problems of transport theory, Soviet Math. Dokl. 37(1988), pp. 497-499. MR947226 (89i:82105)

[4] H. Babovsky, Identification of scattering media from reflected flows, SIAM J. Appl. Math. 51(1991), pp. 1676-1704. MR:1136006 (92k:82059)

[5] G. BAL, Inverse problems for homogeneous transport equations. II. The multidimensional case, Inverse Problems 16(2000), pp. 1013-1028. MR1776480(2001i:85003)

[6] G. Bal And A. Jollivet, Stability estimates in stationary inverse transport, Inverse Probl. Imaging 2(2008), pp. 427-454.

[7] G. BAL, I. LANGmore And F. Monard, Inverse transport with isotropic sources and angularly averaged measurements, Inverse Probl. Imaging 2(2008), pp. 23-42. MR2375321

[8] A. Bondarenko, The structure of the fundamental solution of the time-independent transport equation, J. Math. Anal. Appl. 221(1998), no. 2, 430-451. MR1621726 (99d:82060) 
[9] K. M. Case and P. F. Zweifel, Linear Transport Theory, Addison-Wesley, Reading, MA, 1967. MR0225547(37:1140)

[10] M. Choulli AND P. Stefanov, Inverse scattering and inverse boundary value problems for the linear Boltzmann equation, Comm. P.D.E. 21(1996), pp. 763-785. MR1391523 (97f:35230)

[11] M. Choulli and P. Stefanov, An inverse boundary value problem for the stationary transport equation, Osaka J. Math. 36(1999), pp. 87-104. MR.1670750(2000g:35228)

[12] R. Dautray And J.-L. Lions, Mathematical Analysis and Numerical Methods for Science and Technology. Vol. 6, Springer-Verlag, Berlin, 1993. MR.1295030 (95e:00001)

[13] Y. Kurylev, M. Lassas, and G. Uhlmann, Rigidity of broken geodesic flow and inverse problems, to appear in American Journal of Mathematics.

[14] I. LANGMORE, The stationary transport problem with angularly averaged measurements, Inverse Problems 24(2008), no. 1, 015024 . MR2384783

[15] I. Langmore And S. MCDowall, Optical tomography for variable refractive index with angularly averaged measurements, Comm. PDE 33(2008), pp. 2180-2207.

[16] E. W. LARsen, Solution of multidimensional inverse transport problems, J. Math. Phys. 25(1984), pp. 131-135. MR728896 (85d:82118)

[17] E. W. LARSEN, Solution of three-dimensional inverse transport problems, Transport Theory and Stat. Phys. 17(1988), no. 2-3, pp. 147-167. MR963049 (89i:82108)

[18] N. J. McCormick, Inverse radiative transfer problems: A review, Nucl. Sci. Eng. 112(1992), pp. $185-198$.

[19] S. MCDowALL, An inverse problem for the transport equation in the presence of a Riemannian metric, Pac. J. Math., 216(2004), no. 2, pp. 303-326. MR2094548 (2005h:58029)

[20] S. McDowall, Optical tomography on simple Riemannian surfaces, Comm. P.D.E. 30 (2005), pp. 1379-1400. MR2180309 (2006h:35020)

[21] M. Mokhtar-Kharroubi, Mathematical Topics in Neutron Transport Theory, World Scientific, River Edge, NJ, 1997. MR 1612403(99c:82078)

[22] M. Reed And B. Simon, Methods of Modern Mathematical Physics, Vol. 3, Academic Press, New York, 1979. MR.529429 (80m:81085)

[23] M. Ribarič And I. VidAV, Analytic properties of the inverse $A(z)^{-1}$ of an analytic linear operator valued function A(z), Arch. Rat. Mech. Anal. 32(4)(1969), pp. 298-310. MR0236741 (38:5035)

[24] V. Romanov, Stability estimates in problems of recovering the attenuation coefficient and the scattering indicatrix for the transport equation, J. Inverse Ill-Posed Probl. 4(4)(1966), pp. 297-305. MR.1403885 (97f:35235)

[25] P. Stefanov and G. Uhlmann, Optical tomography in two dimensions, Methods Appl. Anal. 10(2003), pp. 1-9. MR2014159 (2004h:35224)

[26] P. Stefanov and G. Uhlmann, An inverse source problem in optical molecular imaging, Analysis and PDE 1(2008), pp. 115-126. MR2444095

[27] A. TAmasan, An inverse boundary value problem in two-dimensional transport, Inverse Problems 18(2002), pp. 209-219. MR.1893591 (2003h:35283)

[28] _ Optical tomography in weakly anisotropic scattering media, Contemporary Mathematics, vol. 333, Amer. Math. Soc., Providence, RI, 2003, pp. 199-207. MR2032017 (2005a:35284)

[29] J.-N. WANG, Stability estimates of an inverse problem for the stationary transport equation, Ann. Inst. Henri Poincaré 70(1999), pp. 473-495. MR1697917(2000e:82042)

Department of Mathematics, Purdue University, 150 N. University Street, West LAFAYETTE, INDIANA 47907-2067

E-mail address: stefanov@math.purdue.edu

Department of Mathematics, University of Central Florida, 4000 Central Florida Boulevard, Orlando, Florida 32816

E-mail address: tamasan@math.ucf.edu 